\title{
20 Jahre deutsch-russische Zusammenarbeit auf dem Gebiet der pädiatrischen Hämatologie und Onkologie
}

\author{
20 Years of German-Russian Cooperation in Pediatric Hematology and Oncology
}

\section{U. Göbel ${ }^{1}$ \\ U. Creutzig \\ T. Klingebiel ${ }^{3}$}

Institute

${ }^{1}$ Editor Klinische Pädiatrie

${ }^{2}$ Wissenschaftliche

Geschäftsführerin der GPOH

${ }^{3}$ Vorsitzender der GPOH

Bibliografie

DOI http://dx.doi.org/

$10.1055 / \mathrm{s}-0031-1275348$

Klin Padiatr 2011; 223: 107-109

(c) Georg Thieme Verlag KG

Stuttgart · New York

ISSN 0300-8630

Korrespondenzadresse

Prof. Dr. Ulrich Göbel ESPED-Geschäftsstelle

Koordinierungszentrum für

Klinische Studien

Universitätsklinikum

Heinrich-Heine-Universität

Düsseldorf

Moorenstraße 5

40225 Düsseldorf

goebel@arcor.de

\section{Die politische Wende \\ $\nabla$}

Das Jahr 1990 stand im Zeichen der Auflösung der ideologischen, wirtschaftlichen und politischen Systemkonfrontation des „Kalten Krieges“ zwischen Ost und West. Auf der einen Seite standen die Staaten des Warschauer Paktes unter Führung der UdSSR (Union der Sozialistischen Sowjet Republiken) und auf der anderen Seite die NATO (North Atlantic Treaty Organisation) mit den USA als Führungsmacht und den westeuropäischen Staaten als Verbündete.

Vorausgegangen waren 1988 die Wahl von Michail Sergejewitsch Gorbatschow zum Vorsitzenden des Präsidiums des Obersten Sowjets und 1990 seine Wahl zum Staatspräsidenten der UdSSR. Mit seinen epochalen Forderungen nach Glasnost (Transparenz) und Perestroika (Umgestaltung) leitete er eine neue Ära in der UdSSR ein und stellte darüber hinaus vor der 43. UN-Generalversammlung in New York einseitige Abrüstungsschritte in Aussicht. Noch im selben Jahr distanzierte er sich von der Breschnew-Doktrin, mit der der sowjetische Anspruch auf Vorherrschaft innerhalb der Warschauer-Pakt-Staaten festgeschrieben war. Dieser Schritt ermöglichte es den Ländern des Warschauer Pakts, ihre Staatsform selbst zu bestimmen und führte 1989 zu einer Reihe überwiegend friedlicher Revolutionen in Osteuropa. Deutschland konnte seine Wiedervereinigung erlangen. Gorbatschow erhielt 1990 den Friedensnobelpreis [3].

\section{Der Fall des Eisernen Vorhangs und krebskranke Kinder als erste Nutznießer $\nabla$}

Für die deutschen kinderonkologischen Abteilungen bedeuteten diese politischen Umwälzungen die plötzliche Ankunft von krebskranken Kindern aus der UdSSR. Diese kamen nach Deutschland zur Behandlung, da der seit Ende des zweiten Weltkrieges bestehende „Eiserne Vorhang" zwischen Ost und West plötzlich durchlässig war. Vor allem waren es Kinder mit akuter lymphatischer Leukämie (ALL), für die in der UdSSR keine Heilungsaussichten bestanden, während in den westlichen Ländern, z. B. mit den ALL-BFM-Protokollen ca. $80 \%$ der erkrankten Kinder langfristig überlebten. Die unerwartete Einreise der leukämiekranken Kinder, die als erste Privatpersonen vom Fall des Eisernen Vorhangs profitierten, wurde in zahlreichen Nachrichtensendungen im In- und Ausland übertragen.

\author{
Die damalige Behandlungssituation \\ in den deutschen Abteilungen für \\ pädiatrische Hämatologie und Onkologie \\ $\nabla$
}

In den meisten deutschen kinderonkologischen Abteilungen bestand 1990 ein erheblicher Pflegeengpass, sodass der Begriff des Pflegenotstands kursierte. Die Kliniken waren nur bedingt in der Lage, die zusätzlichen Patienten aufzunehmen und zu behandeln. Hinzu kamen die Auswirkungen des Eisernen Vorhangs mit der strikten wirtschaftlichen und kulturellen Trennung. Die unvorbereitet nach Deutschland eingereisten Menschen erlebten zunächst einen Kulturschock. Es fehlte vor allem an sprachlichen Verständigungsmöglichkeiten sowohl für lebenswichtige Belange des alltäglichen Leben wie in der komplexen medizinischen Behandlung. Nur dank der umfassenden Begleitung durch ehrenamtliche Helfer, die meist aus den Elternvereinen für krebskranke Kindern stammten, konnten diese individuellen Notsituationen gemeistert werden.

\section{Der Transfer von Wissen anstelle von Patienten \\ $\nabla$}

In der damaligen Umbruch- und Aufbruchsstimmung entstand der Plan, nicht die Patienten in ein fremdes Land zu transferieren, sondern das Wissen dahin zu bringen, wo die Patienten lebten. Care Deutschland organisierte im Auftrag und mit Unterstützung der deutschen Bundesregierung Symposien für die Ärzte in der inzwischen zur Gemeinschaft unabhängiger Staaten mutierten ehemaligen UdSSR in Minsk, Moskau und St. Petersburg, an denen alle Studienleiter und Leistungserbringer der GPOH teilnahmen und Vorträge hielten. Es wurden 12 Zentren von Minsk im Westen über Moskau im Zentrum bis Wladiwostok im Osten apparativ ausgestattet und die dortigen Ärzte zur Fortbildung nach Deutschland eingeladen. Besondere Verdienste hatte sich damals Herr PD Dr. V. Gerein als russisch sprechender Arzt und Organisator erworben. Kurze Zeit später kam als 13. Zentrum Perm als östlichste Millionenstadt Europas hinzu, da hier die Situation besonders desolat war. Auslösend hierfür war der Besuch des damaligen ARDKorrespondenten Fritz Pleitgen während seiner Reportage über die Verhältnisse in der ehemaligen UdSSR im Dezember 1991 [10] und die gemeinsame Spendenaktion „Ein Herz für Russland“ von ARD und Bild-Zeitung. Hieraus entwickelte sich eine nachhaltige Kooperation mit 
Gründung des gemeinnützigen Vereins „Die Kinder von PermSpendenaktion des WDR“ [4].

\section{Beispielhafte Leistungen der GPOH $\nabla$}

Im Rahmen dieses Editorials können nicht alle Persönlichkeiten genannt werden, die sich in den vergangenen 20 Jahren große Verdienste bei der deutsch-russischen Zusammenarbeit auf dem Gebiet der Kinderonkologie erworben haben. Stellvertretend sind jedoch einzelne Projekte zu nennen, die den umfassenden Rahmen der Zusammenarbeit erkennbar machen.

Als langjähriger GPO-Vorsitzender hat Herr Professor Kornhuber speziell die Bildung der pädiatrischen onko-, hämatologischen Zentren gefördert. Herr Professor Schellong organisierte flankierend spezielle Fortbildungen in Deutschland für besonders qualifizierte russische Ärztinnen und Ärzte zum Erwerb von Spezialkenntnissen. Herr Professor Lampert etablierte die Zytogenetik in Jekaterinburg; Frau Professor Janka förderte die hämatopoetische Stammzelltransplantation in St. Petersburg. Herr Professor Henze hat gemeinsam mit Herrn Professor Rumyancev und Herrn Professor Karaschunski das Moskau-Berlin (MB) Protokoll zur Behandlung der ALL entwickelt und in der Russischen Föderation als prospektiv randomisierte multizentrische Studie mit großem Erfolg eingeführt. Mit der gut praktikablen Therapie erlangen inzwischen $80 \%$ der russischen Kinder mit ALL eine Langzeitremission [6,7]. Herr Professor Harms vermittelt mit gleichbleibendem Elan seit Beginn und bis heute als Kinderpathologe die differenzierte Diagnostik der gut- und bösartigen Tumoren des Kindes- und Jugendalters und fördert engagiert die interdisziplinäre Diagnostik und Therapie auf hohem Niveau. Als besondere Leistung ist die Einführung der über Telemedizin gesteuerten Mikroskopie-Konferenz zu nennen. Über große Entfernungen lässt sich damit jederzeit schnell und zuverlässig eine Referenzbegutachtung der Tumoren von Kindern und Jugendlichen realisieren [11]. Schließlich ist durch Herrn Professor Göbel in Perm die Gründung eines klinischen Registers für Keimzelltumoren unterstützt worden, um bei diesen seltenen und heterogenen Tumoren eine standardisierte Beratung überregional zu ermöglichen [9].

\section{Der Petersburger Dialog und die deutsch-russische Modernisierungspartnerschaft}

Parallel zu diesen langjährigen Entwicklungen erfolgte die weitere staatliche Förderung, die dazu führte, dass schrittweise alle Behandlungskosten von den verschiedenen zuständigen Regierungen und Gebietsverwaltungen übernommen wurden. Der sogenannte „Petersburger Dialog“ wurde 2001 als Diskussionsforum für Deutsche und Russen unter der Schirmherrschaft der Staatspräsidenten ins Leben gerufen. Anlässlich der Plenarsitzung des Petersburger Dialogs 2008 in St. Petersburg, an der auch der russische Präsident Dimitri Medwedew, und die deutsche Bundeskanzlerin Dr. Angela Merkel teilnahmen, wurde unter anderem eine „Gemeinsame Erklärung des Petersburger Dialogs zur Gestaltung der Modernisierungspartnerschaft“ unterschrieben und zahlreiche Arbeitsgruppen zu unterschiedlichen Themenbereichen gebildet. Gleichzeitig durchgeführte deutschrussische Regierungskonsultationen führten zum Abschluss eines „Aktionsprogramms zur Intensivierung der Zusammenarbeit auf dem Gebiet des Gesundheitswesens“ zwischen dem
Bundesministerium für Gesundheit der Bundesrepublik Deutschland und dem Ministerium für Gesundheit und soziale Entwicklung der Russischen Föderation, das von der damaligen Bundesgesundheitsministerin Ulla Schmidt und ihrer russischen Amtskollegin Tatjana Golikowa unterzeichnet wurde [1].

\section{Das Föderale Wissenschaftliche Zentrum für Kinderhämatologie, Onkologie und Immunologie in Moskau $\nabla$}

Unter den verschiedenen Teilprojekten der Gesundheitsvorsorge wird die Kinderonkologie immer an führender Stelle genannt. Für diesen Bereich wurde mit Unterstützung der deutschen Botschaft die Kontakte von Herrn Professor Bergmann zu Professor Rumyancev intensiviert und durch ein vom deutschen Auswärtigen Amt finanzierten Aus- und Weiterbildungsprogramm des Petersburger Dialogs unterlegt. Als ein weiteres Ergebnis des Petersburger Dialogs und der Modernisierungspartnerschaft ist der Bau des Föderalen Wissenschaftlichen Zentrums für Kinderhämatologie, Onkologie und Immunologie in Moskau (FNKZ DGOI Moskau) zu nennen, das von Herrn Professor Rumyancev und Frau Dr. Novichkova beantragt wurde. Der Vertrag für den Bau wurde gemeinsam mit dem Vertrag zur Ostsee-Pipeline von dem damaligen Präsidenten Wladimir Putin und dem damaligen Bundeskanzler Dr. Gerhard Schröder unterzeichnet. Vorausgegangen war der private Besuch des russischen Staatsoberhauptes bei einem 12-jährigen Jungen mit AML, der mehrere Therapien ohne Heilerfolg durchgemacht hatte. Begleitet und unterstützt wird diese Entwicklung durch eine sehr aktive Bürgerinitiative in Moskau [12].

Dieses Zentrum wird durch eine in Koblenz ansässige Firma mit langjähriger Erfahrung im Krankenhausbau errichtet und ist für 250 Betten und ein großes Ambulatorium geplant (s. Abb. 1) [14]. Nach endgültiger Fertigstellung werden dort 400 Ärzte und Wissenschaftler und 900 weitere Mitarbeiter in 30 Abteilungen und Stationen tätig sein. Als spezielle Einrichtungen sind u. a. ein Operationstrakt mit 4 Operationssälen, Kinderpathologie, Radiologische Diagnostik, Strahlentherapie, Nuklearmedizin, Transfusionsmedizin mit Immungenetik, Zytogenetik und Molekularbiologie zu nennen. Geplant ist ein Comprehensive Cancer Cen-

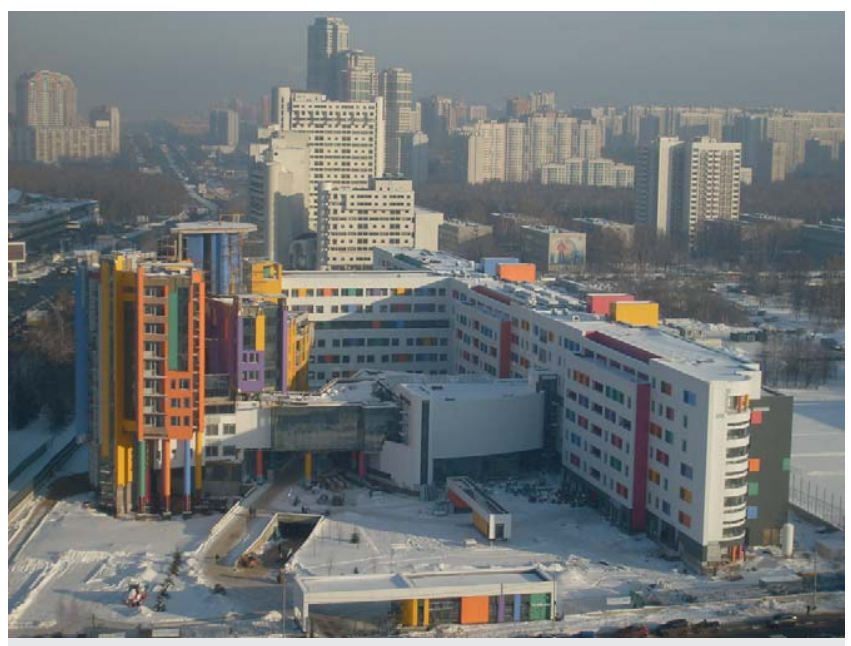

Abb. 1 Das im Bau befindliche Föderale Wissenschaftliche Zentrum für Kinderhämatologie, Onkologie und Immunologie in Moskau (Eingangsansicht mit Elternhotel im Vordergrund, links, Januar 2011). 
ter für Kinder und Jugendliche nach modernstem westlichem Standard, das die Diagnostik und Therapie gleichermaßen wie die klinische und experimentelle Forschung sicherstellen soll. Auch wenn beim Aufbau des Zentrums zuerst die häufigeren Erkrankungen im Blickpunkt stehen, werden langfristig auch die seltenen Erkrankungen im Sinne der Patientengerechtigkeit berücksichtigt werden [8].

Zusätzliche Gebäude bereichern den Krankenhauskomplex: Ein Lehrtrakt mit Wohnräumen für Gastärzte wird die wissenschaftliche Kommunikation im internationalen Rahmen ermöglichen. Ebenso erlaubt ein eigenes Elternhotel die Behandlung von Problempatienten aus der ganzen Russischen Föderation unter patientenfreundlichen Rahmenbedingungen.

\section{Vernetzung und Internetportal}

\section{$\nabla$}

Die GPOH-Geschäftsführerin hat im Rahmen des Arbeitstreffens im Februar 2011 Erfahrungen über weitreichende Kooperationen und Studieninfrastrukturen der GPOH an die Moskauer Kinderhämatologen/-onkologen weitergegeben. Dies betraf insbesondere die im Rahmen des Kompetenznetzes Pädiatrische Onkologie und Hämatologie etablierten Strukturen, z.B. zur Dokumentation und zur Referenzdiagnostik [2].

Der Aufbau eines Internetportals mit entsprechenden Patientenund Elterninformationen in russischer Sprache ist in Planung.

\section{Wissenschaftlicher Beirat}

$\nabla$

Ein so großes Zentrum wie das FNKZ DGOI Moskau wird schrittweise in Nutzung genommen werden, selbst wenn es, wie geplant am 1.6.2011, der Öffentlichkeit als eröffnet vorgestellt wird. Zur Beratung und Begleitung dieser Inbetriebnahme ist ein internationaler wissenschaftlicher Beirat durch Herrn Professor Rumyancev berufen worden, dem Wissenschaftler aus Deutschland, Frankreich, Israel, Italien, Österreich und der Schweiz angehören. Aus den dargestellten historischen Gründen haben bisher die GPOH-Mitglieder eine dominierende Rolle, die sich jedoch bei der zeitlich befristeten Berufung auf 2 Jahre rasch ändern wird. Die Mitglieder des Beirates werden auf Anfrage aktiv. Aktuell findet für ausgewählte russische Ärztinnen und Ärzte, die in dem neuen Zentrum leitende Funktionen übernehmen werden, ein Hospitationsprogramm in Deutschland statt. Dieses Hospitationsprogramm wird vor allem durch die großzügige Unterstützung der TRANSAID-Stiftung [13], aber auch durch die Förderung des Auswärtigen Amtes/Petersburger Dialog möglich.

Nach den praktischen Aspekten der medizinischen Versorgung, die jetzt im Vordergrund stehen, werden schon bald die klinischen Studien und die Grundlagenforschung in den Blickpunkt rücken. Hierfür sind weiterhin Ressourcen und gesetzliche Initiativen notwendig [8].

Dringlich ist beispielsweise eine Gesetz, das den Aufbau eines epidemiologischen Krebsregisters erlaubt und diagnosebezogene klinische Register langfristig positioniert. Dies sind wesentliche Voraussetzungen für die Etablierung von Therapieoptimierungsstudien und den weiteren Aufbau eines interdisziplinären Netzwerkes für krebskranke Kinder in der Russischen Föderation [5].

\section{Langzeitperspektiven}

\section{$\nabla$}

Mit dem neuen Zentrum wird ein Vorbild für die ganze Russische Föderation geschaffen. Es ist zu hoffen, dass dadurch auch weitere Verbesserungen bei den peripheren Zentren folgen, um eine flächendeckende Versorgung der Kinder und Jugendlichen mit gut- und bösartigen Blut- und Tumorerkrankungen auf hohem Niveau sicherzustellen. Die positiven Erfahrungen mit der MB-Leukämiestudie werden rasch zu internationalen Kooperationen führen $[6,7]$.

Dem neuen Zentrum ist zu wünschen, dass es richtungsweisende Impulse setzen kann, sowohl von seiner Größe her als auch seiner Einbindung in Russland und Europa.

Interessenkonflikt: Die Autoren erklären hiermit, dass kein Interessenkonflikt besteht.

\section{Literatur}

1 Bergmann W, Krawietz W, Hrsg. Nach 20 Jahren Perestrojka. Wege zu einer Neuen Weltordnung. Michail S. Gorbatschow und Richard von Weizsäcker im Gespräch auf dem Petersberg in Bonn. Abschlussberichte des Petersburger Dialoges (Jahrgänge 2008 und 2009) herausgegeben vom Petersburger Dialog e.V., Berlin. (Rechtstheorie. Zeitschrift für Logik und Juristische Methodenlehre, Rechtsinformatik, Kommunikationsforschung, Normen- und Handlungstheorie, Soziologie und Philosophie des Rechts. 2009, Band 40, Heft 2), Duncker \& Humblot, Berlin; 2009, ISBN 978-3-428-13236-2

2 Creutzig U, Herold R, Henze G. Ergebnisse des Kompetenznetzes Pädiatrische Onkologie und Hämatologie - ein Rückblick. Klin Padiatr 2010; 222: 333-336

3 Dalos G. Gorbatschow. Mensch und Macht. Eine Biografie. C. H. Beck, München 2011, ISBN 978-3-406-61340-1

4 Die Kinder von Perm/Spendenaktion des Westdeutschen Rundfunks e.V. Hrsg. Den Kindern eine Hoffnung geben - 10 Jahre Kinderkrebsklinik Perm Köln 2006

5 Göbel U, Kontny U, Gortner L et al. Netzwerke in der Pädiatrie aus Sicht der Klinischen Pädiatrie. Klin Padiatr 2009; 221: 131-133

6 Karachunski A, Herold R, von Stackelberg A et al. Results of the first randomized multicentre trial on childhood acute lymphoblastic leukaemia in Russia. Leukemia. 2008; 22: 1144-1153

7 Karachunski A, Miakova NV, Rumiantseva IV et al. The results of a multicenter trial of acute lymphoblastic leukemia treatment on ALLMB 91/ALL-BFM 90m in children: analysis of efficacy and toxicity. Ter Arkh 2007; 79: 19-26

8 Klingebiel T, Creutzig U, Christaras A et al. Meilensteine der Gesundheitspolitik und seltene Erkrankungen. Klin Padiatr 2010;222: 121-123

9 Mushinskaya MV, Ryskal OV, Sokolev YY et al. Management of children with germ cell tumors in the Perm territory. Pediatr Haematol Oncol Immunopathol 2009; 8: 18-24

10 Pleitgen F. ARD-Brennpunkt „Ein Herz für Russland - Perm in Not“. Gesendet am 04.12.1991

11 Ryskal O, Muschinskaja M, Göbel U et al. Telemicroscopic conferences for children of the Perm territory with suspected or proven malignant solid tumors. Klin Padiatr 2010; 222: 199-202

12 Schenke Leben. Stiftung zur Unterstützung von Kindern mit onkologischen, hämatologischen und anderen schweren Erkrankungen. Gegründet 2006 von den russischen Schauspielerinnen: Chulpan Hamatova und Dina Korzun Moskau 2006

13 TRANSAID-Stiftung für Krebskranke Kinder, Hrsg. - ein Leben retten, das noch ganz am Anfang steht - Koblenz 2007

14 TRANSUMED Koblenz, Hrsg. Föderales Wissenschaftlich-Klinisches Zentrum für Kinderhämatologie. Onkologie und Immunologie in Moskau. Leninskij Prospekt. Koblenz; 2006 